\title{
Quantification of Electrochemical Nanoscale Processes in Lithium Batteries by Operando ec-(S)TEM
}

\author{
B. L. Mehdi ${ }^{1}$, J. Qian ${ }^{2}$, E. Nasybulin ${ }^{2}$, C. Park ${ }^{3}$, D. A. Welch ${ }^{4}$, R. Faller ${ }^{4}$, H. Mehta ${ }^{5}$, W. A. Henderson ${ }^{2}$, \\ W. Xu ${ }^{2}$, C. M.Wang ${ }^{5}$, J. E. Evans ${ }^{5}$, J. Liu ${ }^{2}$, J. -G. Zhang ${ }^{2}$, K. T. Mueller ${ }^{5,6}$, N. D. Browning ${ }^{1}$ \\ ${ }^{1}$ Joint Center for Energy Storage Research, Fundamental and Computational Science Directorate, \\ PNNL, Richland, WA, USA \\ ${ }^{2}$ Joint Center for Energy Storage Research, Energy and Environmental Directorate, PNNL, Richland, \\ WA, USA \\ ${ }^{3}$ Department of Industrial and Manufacturing Engineering, Florida State University, Tallahassee, FL, \\ USA \\ ${ }^{4}$ Department of Chemical Engineering and Materials Science, University of California, Davis, CA, USA \\ ${ }^{5}$ Joint Center for Energy Storage Research, Environmental Molecular Sciences Laboratory, PNNL, \\ Richland, WA, USA \\ ${ }^{6}$ Department of Chemistry, Penn State University, University Park, PA, USA
}

Lithium (Li)-ion batteries are currently used for a wide variety of portable electronic devices, electric vehicles and renewable energy applications [1,2]. In addition, extensive worldwide research efforts are now being devoted to more advanced "beyond Li-ion" battery chemistries - such as lithium-sulfur (Li-S) [3] and lithium-air $\left(\mathrm{Li}-\mathrm{O}_{2}\right)$ [4] - in which the carbon anode is replaced with Li metal. However, the practical application of Li metal anode systems has been highly problematic. The main challenges involve controlling the formation of a solid-electrolyte interphase (SEI) layer and the suppression of Li dendrite growth during the charge/discharge process (achieving "dendrite-free" cycling). The SEI layer formation continuously consumes the electrolyte components creating highly resistive layer, which leads to the rapid decrease of cycling performance and degradation of the $\mathrm{Li}$ anode [5]. The growth of $\mathrm{Li}$ metal dendrites at the anode contributes to rapid capacity fading (the presence of "dead Li" created during the discharge leads to an increased overpotential on charging) and, in the case of continuous growth, leads to internal short circuits (the dendrites contact the cathode) and extreme safety issues [6].

Here we demonstrate the application of an operando electrochemical scanning transmission electron microscopy (ec-(S)TEM) cell to study the SEI layer formation and the initial stages of Li dendrite growth - the goal is to develop a mechanism for mitigating the degradation processes and increasing safety. Bright field (BF) STEM images in Figure 1 A-C show Li metal deposition and dissolution processes at the interface between the Pt working electrode and the lithium hexafluorophosphate $\left(\mathrm{LiPF}_{6}\right)$ in propylene carbonate (PC) electrolyte during 3 charge/discharge cycles. A contrast reversal caused by Li metal being lighter/less dense than surrounding electrolyte ( $\mathrm{Li}$ appears brighter than the background in BF STEM images) allows Li to be uniquely identified from the other components in the system - the only solid material that is less dense than the electrolyte is Li metal. Using these images, we can precisely quantify the total volume of Li deposition, the thickness of the SEI layer (observed as a ring of positive contrast around the electrode) and alloy formation due to $\mathrm{Li}^{+}$ion insertion during each cycle. Furthermore, at the end of each discharge cycle we can quantify the presence of "dead Li" detached from the $\mathrm{Pt}$ electrode, thereby demonstrating the degree of irreversibility (and degradation of $\mathrm{Pt}$ electrode) associated with insertion/removal of $\mathrm{Li}^{+}$during this process. Such analyses provide significant insights into Li metal dendrite growth, which is critical to understand the complex interfacial reactions needed to be controlled for future Li-based and next generation energy storage systems. [7] 
References:

[1] J. M. Tarascon, M. Armand, Nature, 414, (2001), 414, 359-367

[2] J. B. Goodenough, Y. Kim, Chem. Mater.,22, (2010), 587-603

[3] X. L. Jie, L. F. Nazar, J. Mater. Chem., 20, (2010), 9821-9826

[4] P.G. Bruce, S. A. Freunberger, L. J. Hardwick, J. M. Tarascon, Nat. Mater., 11, (2012), 19-29

[5] P. Verma, P. Maire, P. Novak, Electrochim. Acta, 55, (2010), 6332-6341

[6] J. Wen, Y. Yu, C. Chen, Mater. Express, 2, (2012), 197-212

[7] This work was primarily supported by JCESR, an Energy Innovation Hub funded by DOE-BES. The development of the operando stage was supported by the Chemical Imaging LDRD Initiative at PNNL. PNNL is a multi-program national laboratory operated by Battelle for the U.S. DOE under Contract DEAC05-76RL01830. A portion of the research was performed at the EMSL user facility sponsored by DOE-BER and located at PNNL. The multi-target tracking algorithm is supported by NSF-1334012.
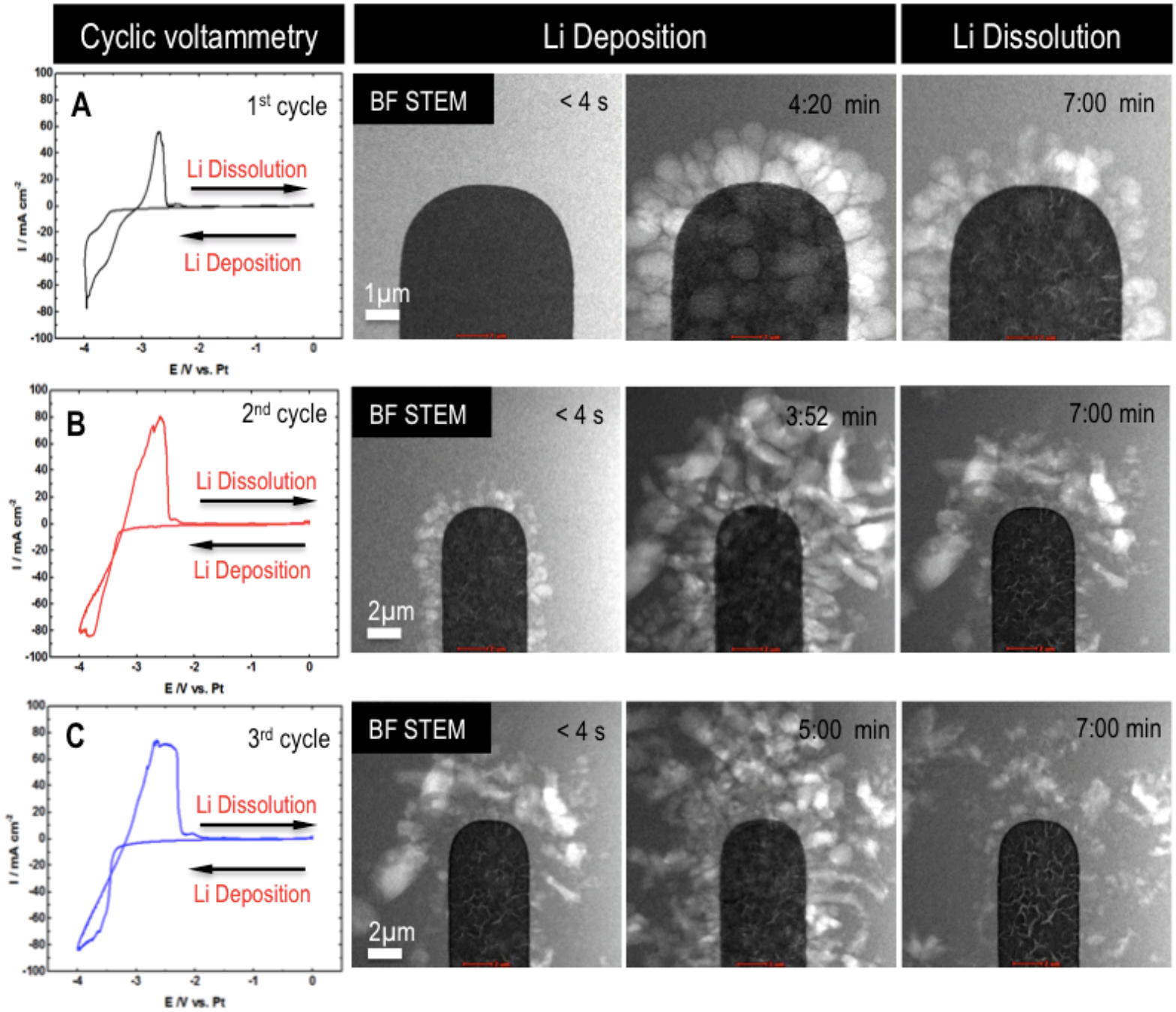

Figure 1. Cyclic voltammetry and BF STEM images of Li deposition and dissolution at the interface between the $\mathrm{Pt}$ working electrode and the $\mathrm{LiPF}_{6} / \mathrm{PC}$ electrolyte during the (A) first, (B) second and, (C) third charge/discharge cycles of the operando cell. 